\title{
Conjunto de arcos de realimentação sob restrições de forçamento é FPT
}

\author{
Leonardo C. de Abreu ${ }^{1}$, Manoel Campêlo ${ }^{1}$, Ana Karolinna Maia ${ }^{2}$ \\ ${ }^{1}$ Departamento de Estatística e Matemática Aplicada - Universidade Federal do Ceará \\ Campus do Pici, B1. 910, 60.440-900 - Fortaleza - CE - Brazil \\ ${ }^{2}$ Departamento de Computação - Universidade Federal do Ceará \\ Campus do Pici, Bl. 910, 60.440-900 - Fortaleza - CE - Brazil \\ \{leonardo.abreu,mcampelo\}@lia.ufc.br, karolmaia@ufc.br
}

\begin{abstract}
The feedback arc set problem (FAS) consists in finding a minimum size subset of arcs which contains at least one arc of every directed circuit of a directed graph. We study the FAS problem subject to forcing constraints. These constraints state that at least one element of certain pairs of arcs must be included in any feasible solution. It is known that the feedback arc set problem is FPT. We show that the version with forcing constraints is also FPT when parameterized by the size of the solution.
\end{abstract}

Resumo. O problema do conjunto de arcos de realimentação (FAS) consiste em encontrar um subconjunto de arcos de tamanho mínimo que contenha ao menos um elemento de cada circuito direcionado de um grafo direcionado. Estudamos o problema FAS sujeito a restrições de forçamento, as quais determinam que ao menos um elemento de certos pares de arcos deve estar presente em uma solução viável. Sabe-se que o problema de arcos de realimentação é FPT. Mostramos que o problema com restrições de forçamento também é FPT quando parametrizado pelo tamanho da solução.

\section{Introdução}

Restrições disjuntivas positivas, ou de forçamento, determinam que ao menos um elemento de certos pares de elementos deve estar presente em uma solução viável. Essas restrições são convenientemente representadas por um grafo simples chamado grafo de forçamentos. Nesse grafo, os elementos são os vértices, enquanto as arestas representam os forçamentos entre pares de vértices. Portanto, uma solução viável deve cobrir todas as arestas do grafo de forçamentos.

Versões do problema da árvore geradora máxima, do caminho mínimo e do emparelhamento máximo sujeitas a restrições disjuntivas positivas foram introduzidas por [Darmann et al. 2011]. Os autores também mostraram que essas versões são fortemente NP-Difíceis.

Em [Mapa and Urrutia 2015] foi introduzida a versão com restrições disjuntivas positivas para o problema do subgrafo acíclico máximo. Além disso, mostrou-se que determinar se existem soluções viáveis para uma instância do problema é um problema da classe NP-Completo. 
Estudamos o problema do conjunto de arcos de realimentação sujeito a restrições de forçamento (FASFC) - do inglês feedback arc set under forcing constraints, o qual é definido como segue: dados um grafo direcionado $D=(V, A)$ e um grafo simples $G=$ $(A, E)$, encontre um subconjunto $F \subseteq A$ de mínima cardinalidade, tal que $D[A \backslash F]$ é acíclico e $F$ é uma cobertura de vértices em $G$. Quando $E=\emptyset$ temos o conhecido problema do conjunto de arcos de realimentação (FAS).

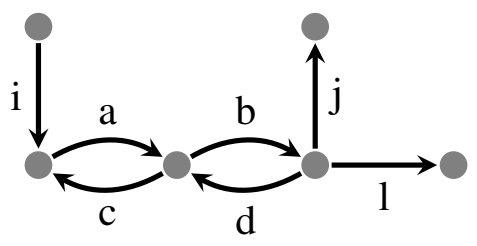

(a) $D=(V, A)$

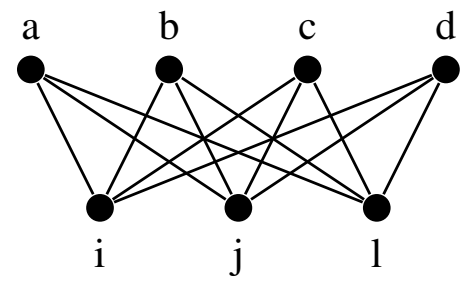

(b) $G=(A, E)$

Figura 1. Exemplo de instância do FASFC.

A Figura 1 apresenta um exemplo de instância do FASFC. A solução ótima dessa instância é o conjunto de $\operatorname{arcos}\{a, b, c, d\}$. Note que se não houvessem as restrições de forçamento, os $\operatorname{arcos} a$ e $b$ formariam um conjunto de arcos de realimentação mínimo. Vale destacar também que a solução ótima dessa instância não contém elementos da cobertura de vértices mínima de $G$.

Sabe-se que o problema do conjunto de arcos de realimentação (FAS) é NP-Difícil [Karp 1972], e, portanto, também a versão com restrições de forçamento. Além disso, o FAS parametrizado pelo tamanho da solução é FPT [Chen et al. 2008], assim como o problema da cobertura de vértices [Mehlhorn 1984, Downey and Fellows 1992]. Mostramos que o FASFC também é FPT quando parametrizado pelo tamanho da solução a partir de uma redução para o problema do conjunto de vértices de realimentação (direcionado).

\section{Redução para o conjunto de vértices de realimentação}

Um problema de minimização é tratável por parâmetro fixo (FPT) - do inglês fixed parameter tractable — , parametrizado pelo valor da solução, se, dada uma instância de tamanho $n$ e um inteiro $k$, existe um algoritmo que encontra uma solução de valor no máximo $k$ em tempo $f(k) n^{O(1)}$.

O trabalho de [Chen et al. 2008] apresenta um algoritmo FPT de tempo $4^{k} k ! n n^{O(1)}$ para o problema do conjunto de vértices de realimentação (DFVS) - do inglês directed feedback vertex set. Esse problema é similar ao FAS, entretanto, o objetivo é encontrar um conjunto de vértices cuja remoção deixa o grafo direcionado acíclico. Mostraremos a seguir que o problema do conjunto de arcos de realimentação sujeito a restrições de forçamento também é FPT.

Para isso, adaptamos o resultado de [Even et al. 1998] para a versão com restrições de forçamento. Esse resultado afirma que um grafo direcionado $D$ possui um conjunto de arcos de realimentação de cardinalidade no máximo $k$ se, e somente se, o grafo linha direcionado de $D$ possui um conjunto de vértices de realimentação de cardinalidade menor ou igual a $k$. A partir disso, obtemos uma redução parametrizada do problema FASFC para o DFVS. 
O FAS sob restrições de forçamento parametrizado pelo tamanho da solução ( $k$-FASFC) é definido como: dados um grafo direcionado $D=(V, A)$, o grafo de forçamento $G=(A, E)$ e um inteiro positivo $k$, encontre um conjunto de $\operatorname{arcos} F \subseteq A$, tal que $D[A \backslash F]$ é acíclico, $F$ é cobertura de vértices em $G$ e $|F| \leq k$.

Teorema 1. O problema k-FASFC é FPT.

Demonstração. Seja $(D, G, k)$ uma instância do $k$-FASFC. Construiremos um grafo direcionado $D^{\prime}$, tal que todo arco de $D$ tem um vértice correspondente em $D^{\prime}$ e $D^{\prime}$ possui um conjunto de vértices de realimentação de cardinalidade no máximo $k$ se, e somente se, $D$ possui um conjunto de arcos de realimentação que cobre todas as arestas de $G$ de tamanho no máximo $k$ (note que os arcos de $D$ são vértices em $G$ ).

O grafo linha direcionado $L$ de $D$ é o grafo direcionado tal que $V(L)=A(D)$ e, dados $a, b$ arcos de $D,(a, b) \in A(L)$ se, e somente se, a cabeça de $a$ coincide com a cauda de $b$.

Faça $D^{\prime}$ igual ao grafo linha direcionado de $D$. Em seguida, para toda aresta em $G$, adicione em $D^{\prime}$ um 2-ciclo entre os vértices correspondentes as extremidades da aresta. Caso já hajam arcos entre os vértices, adicione nenhum ou apenas o arco faltante para formar o 2-ciclo. Esse processo pode ser realizado, no pior caso, em tempo $O\left(|A|^{2}\right)$. Além disso, obtemos uma instância $\left(D^{\prime}, k\right)$ para o FVS que preserva o parâmetro $k$, e $\left|V\left(D^{\prime}\right)\right|=|A(D)|$.

Seja $F \subseteq A(D)$ solução para o $k$-FASFC com $|F| \leq k$. Defina $F^{\prime} \subseteq V\left(D^{\prime}\right)$ o conjunto de vértices em $D^{\prime}$ correspondentes aos arcos de $F$. Pela construção de $D^{\prime}, F^{\prime}$ cobre todos os circuitos direcionados originalmente presentes no grafo linha direcionado de $D$. Além disso, como $F$ é uma cobertura de vértices de $G, F^{\prime}$ cobre todos os 2-ciclos de $D^{\prime}$ correspondentes as arestas de $G$.

Sejam $F^{\prime} \subseteq V\left(D^{\prime}\right)$, com $\left|F^{\prime}\right| \leq k$, um conjunto de vértices de realimentação de $D^{\prime}$ e $F \subseteq A(D)$ o conjunto de arcos de $D$ que corresponde a $F^{\prime}$. Como $F^{\prime}$ cobre todo 2-ciclo de $D^{\prime}, F$ cobre todas as arestas de $G$. Considere agora um circuito direcionado $C$ qualquer de $D$. Observe que existe um circuito correspondente em $D^{\prime}$ que é coberto por um vértice em $F^{\prime}, \operatorname{logo} F$ contém pelo menos um arco de $C$.

Nos dois casos acima, $|F|=\left|F^{\prime}\right|$. Como DFVS é FPT no tamanho da solução, o $k$-FASFC é FPT.

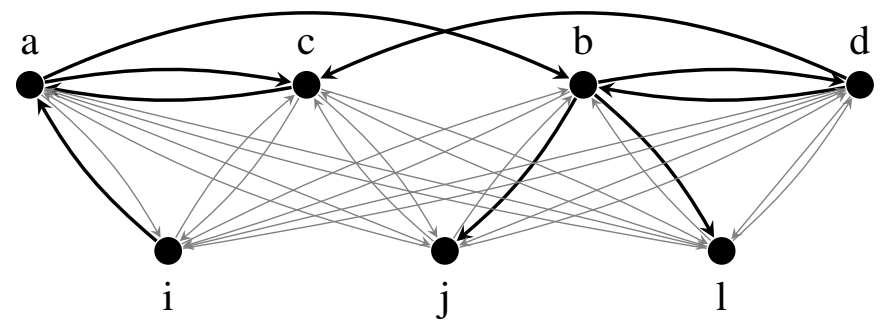

Figura 2. Resultado da redução do exemplo da Figura 1 e parâmetro $1 \leq k \leq 7$

A Figura 2 apresenta um exemplo da redução apresentada no Teorema 1 aplicada aos grafos da Figura 1 e parâmetro $1 \leq k \leq 7$. 
A redução apresentada na prova do teorema 1 não mostra apenas que o FASFC é FPT, mas também permite adaptar resultados de complexidade parametrizada do FVS para o FASFC de modo que o fator assintótico é mantido.

Corolário 1.1. $k$-FASFC possui algoritmo de tempo $O\left(4^{k} k ! \cdot|E(D)|^{c}\right)$, com c constante.

Demonstração. O resultado deduz-se imediatamente a partir da redução do Teorema $1 \mathrm{e}$ do algoritmo de [Chen et al. 2008] de tempo $4^{k} k ! n^{O(1)}$ para o FVS.

\section{Considerações finais}

Adaptamos o resultado de [Even et al. 1998] para produzir um algoritmo FPT para o $k$-FASFC. O resultado também permite obter algoritmos parametrizados para o problema a partir de algoritmos para o DFVS. Como o DFVS é equivalente ao FAS, algoritmos melhores para o problema clássico se traduzem em algoritmos melhores para o problema com forçamentos. É possível utilizar a técnica de árvore de busca limitada para obter um resultado semelhante, que pode ser generalizado para outros problemas com restrições de forçamento. Entretanto o tempo assintótico aumenta por um fator de $2^{k-1}$ no pior caso.

Sabe-se que o FAS com restrições disjuntivas negativas é uma generalização do problema do conjunto independente máximo, que é $W$-Hard para classes gerais de grafos. Entretanto, questiona-se para quais classes esse problema possui algoritmo FPT, pois é sabido que diversos problemas $W$-Hard são FPT para classes de grafos livres de ciclos pequenos [Raman and Saurabh 2008].

\section{Referências}

Chen, J., Liu, Y., Lu, S., O’Sullivan, B., and Razgon, I. (2008). A fixed-parameter algorithm for the directed feedback vertex set problem. J. ACM, 55.

Darmann, A., Pferschy, U., Schauer, J., and Woeginger, G. J. (2011). Paths, trees and matchings under disjunctive constraints. Discrete Applied Mathematics, 159(16):17261735. CTW 2009.

Downey, R. and Fellows, M. (1992). Fixed parameter tractability and completeness. In Congressus Numerantium, volume 87, pages 191-225.

Even, G., Naor, J., and Schieber, B. (1998). Approximating minimum feedback sets and multicuts in directed graphs. Algorithmica, 20:151-174.

Karp, R. (1972). Reducibility among combinatorial problems. In Complexity of Computer Computations, volume 40, pages 85-103.

Mapa, S. M. S. and Urrutia, S. (2015). On the maximum acyclic subgraph problem under disjunctive constraints. Information Processing Letters, 115(2):119-124.

Mehlhorn, K. (1984). Data structures and algorithms 2. In Brauer, W., Rozenberg, G., and Salomaa, A., editors, Graph Algorithms and NP-Completeness, Berlin. Springer.

Raman, V. and Saurabh, S. (2008). Short cycles make W-Hard problems hard: FPT algorithms for W-Hard problems in graphs with no short cycles. Algorithmica, 52(2):203-225.

Nós gostaríamos de agradecer aos revisores anônimos pelas sugestões e comentários importantes. 